\title{
Haploidentical CD19/CD22 bispecific CAR-T cells induced MRD-negative remission in a patient with relapsed and refractory adult B-ALL after haploidentical hematopoietic stem cell transplantation
}

\author{
Hejin Jia ${ }^{1+}$, Zhenguang Wang ${ }^{1+}$, Yao Wang ${ }^{1+}$, Yang Liu ${ }^{2}$, Hanren Dai ${ }^{1}$, Chuan Tong ${ }^{1}$, Yelei Guo ${ }^{1}$, Bo Guo ${ }^{2}$, \\ Dongdong $\mathrm{Ti}^{1}$, Xiao Han ${ }^{1}$, Qingming Yang ${ }^{1}$, Zhiqiang $\mathrm{Wu}^{1 *}$ and Weidong $\mathrm{Han}^{1 *}$
}

\begin{abstract}
Background: Chimeric antigen receptor T (CAR-T) cell therapy simultaneously against CD19 and CD22 is an attractive strategy to address the antigen escape relapse after CD19-directed CAR-T cell therapies. However, the potential of optimizing the durability of remission by this approach in patients with B cell acute lymphoblastic leukemia (B-ALL) remains a critical unanswered question so far.

Case presentation: We treated an adult patient with relapsed and refractory B-ALL after haploidentical hematopoietic stem cell transplantation (HSCT) by administering haploidentical CAR-T cells targeting both CD19 and CD22 following preparative lymphodepleting chemotherapy. This patient has remained in minimal residual disease-negative remission for more than 14 months and has been tapered off graft versus host disease prophylaxis.
\end{abstract}

Conclusions: CAR simultaneously targeting CD19 and CD22 has the potential of inducing long-term remission in patients with B-ALL.

Keywords: Chimeric antigen receptor, CAR-T, Bispecific CAR-T, GVHD, Haploidentical CAR-T

\section{Background}

CD19-directed chimeric antigen receptor T (CAR-T) cells have shown unprecedented initial response rates in relapsed/refractory (R/R) B cell acute lymphoblastic leukemia (B-ALL); however, relapse due to the loss or downregulation of the CD19 is an emerging threat to this innovative form of cellular immunotherapy $[1,2]$. CAR-T cells specific for $\mathrm{CD} 22$, another $\mathrm{B}$ cell lineage of antigen, have also shown comparable potency to CD19-directed CAR-T cells in 21 adult patients with B-ALL [3]. CAR-T cells simultaneously targeting CD19 and CD22 have demonstrated potential benefit of overcoming CD19 immune escape [3],

\footnotetext{
*Correspondence: 43713130@qq.com; hanwdrsw69@yahoo.com

${ }^{+}$Hejin Jia, Zhenguang Wang and Yao Wang contributed equally to this work. ${ }^{1}$ Molecular \& Immunological Department, Bio-therapeutic Department, Chinese PLA General Hospital, No. 28 Fuxing Road, Beijing 100853, China Full list of author information is available at the end of the article
}

and early clinical experience with this approach in pediatric and adult $B$ cell malignancies has shown promising results [4-7], but the effect of this approach on long-term disease control either in the autologous or in the allogeneic setting remains a critical unanswered question so far.

Currently, CD19-directed CAR-T cells are mainly manufactured from patient-derived T cells. However, in some circumstances such as failure of autologous CAR$\mathrm{T}$ cell manufacturing or without time window for leukapheresis because of the active disease, CAR-T cells are also generated from donor-derived $\mathrm{T}$ cells [8-11]. $\mathrm{Cu}-$ mulative data from the clinical trials of donor-derived CAR-T cells have shown that donor-derived CAR-T cells targeting CD19 could effectively salvage relapsed B-ALL after allogeneic hematopoietic stem cell transplantation (HSCT) with a lesser risk of graft versus host disease (GVHD) flare [11-13]. 
We have designed a bispecific CAR simultaneously targeting both CD19 and CD22 (TanCAR-19/22) and initiated a clinical trial exploring $\mathrm{T}$ cells expressing this CAR (TanCAR-T 19/22 cells) in R/R B cell malignancies. Here, we report on the immunologic and long-term clinical effects of this haploidentical (haplo) TanCAR-T 19/ 22 cells used in a compassionate use setting in a patient with relapsed and refractory adult B-ALL after haploHSCT. As of 28 March 2019, the patient has remained in minimal residual disease (MRD)-negative remission for more than 14 months.

\section{Case presentation}

This subject was a 22-year-old man with B-ALL who had third bone marrow (BM) relapse before enrollment on to our compassionate clinical protocol using TanCAR-T 19/ 22 cells. He was diagnosed with B-ALL with more than $100 \times 10^{9} / \mathrm{L}$ WBC count and normal karyotype in January 2016. After complete remission (CR) 2 , he underwent haplo-HSCT from his father 10 months after the original diagnosis. He had suffered hemorrhagic cystitis and stage 1 gastrointestinal acute GVHD within 2 months post haploHSCT, which resolved with 15 daily doses of methylprednisolone $50 \mathrm{mg}$ followed by 5 daily doses of methylprednisolone $100 \mathrm{mg}$. Three months after discontinuation of the cyclosporine A and methylprednisolone, his disease relapsed with $6.4 \%$ marrow blasts when he still had full donor chimerism, then rapidly progressed with $56.5 \%$ marrow blasts by flow cytometry 10.6 months post haploHSCT, and undetectable donor chimerism was noted at the same time. He received salvage chemotherapy with MOEP ( 3 daily doses of mitoxantrone $10 \mathrm{mg}$, vindesine 4 $\mathrm{mg}, 3$ daily doses of etoposide $100 \mathrm{mg}$, and 5 daily doses of dexamethasone $15 \mathrm{mg}$ ) and had severe bone marrow depression and no response with $65.4 \%$ marrow blasts 1 month after the first cycle of MOEP. Then, he was treated on our haplo-CAR-T 19 cell protocol. He received cytoreduction chemotherapy with vindesine and methylprednisolone plus hydroxyurea and lymphodepleting therapy with daunorubicin and cyclophosphamide, and his marrow blasts dropped to $12.7 \%$ prior to haplo-CAR-T 19 cell infusion. Haplo-CAR-T 19 cells at a dose of $4.91 \times 10^{6} / \mathrm{kg}$ $\left(2.89 \times 10^{7} \mathrm{~T}\right.$ cells $/ \mathrm{kg}, 17 \%$ transfection efficiency) were administered and induced MRD-negative CR (MRD-CR) and full donor chimerism within 2 weeks after infusion. The infused haplo-CAR-T 19 cells exhibited rapid expansion and peaked with 15,281 copies per microgram DNA within the first 2 days after infusion, but dropped from 3374 copies per microgram DNA at day 7 to 468 copies per microgram DNA at day 12; methylprednisolone $160 \mathrm{mg}$ and dexamethasone $5 \mathrm{mg}$ were used at day 11 for treatment of the infusion-related grade 3 cytokine release syndrome (CRS). He experienced stage 3 skin acute GVHD within 1 month after haplo-CAR-T 19 cell infusion, which was under control with 5 daily doses of methylprednisolone $40 \mathrm{mg}$ plus cyclosporin A $80 \mathrm{mg}$ administered from day 31 after haplo-CAR-T 19 cell infusion. However, 1 month after obtaining MRD-CR, his disease exhibited florid progression with WBC count increasing from $1.59 \times 10^{9}$ to $12.52 \times 10^{9} / \mathrm{L}$ and corresponding percentage of circulating blasts increasing from 1.39 to $67.37 \%$ within 2 weeks; his bone marrow exhibited highly active cellular proliferation with $59.67 \%$ blasts that had the expression pattern CD19+ $\mathrm{CD} 34+\mathrm{CD} 10+\mathrm{CD} 22+\mathrm{CD} 38+\mathrm{CD} 58+\mathrm{CD} 33+\mathrm{CD} 20-$ CD13- CD15-. At the same time, undetectable haploCAR-T 19 cells and donor chimerism were documented.

In this case, other therapies including TanCAR-T 19/ 22 cells rather than salvage chemotherapy or reinfusion of CAR-T 19 cells could be a potential treatment option for this patient due to the poor response to salvage chemotherapy and poor persistence of infused CAR-T 19 cells. However, higher tumor burden and short-term interval post discontinuation of steroid greatly increased the risk of failure of the generation of autologous CAR$T$ cells; florid progression of the disease made waiting till steroid tapered off was less feasible. Donor-derived TanCAR-T 19/22 cell therapy was an optimal approach to overcome this problem, but as well known, haploCAR-T cell therapies were not to be advocated routinely in the setting of prior GVHD requiring steroid mainly due to raised concern for the high risk of GVHD reactivation. After more careful consideration of the clinical benefits and risks of the second haplo-CAR-T cell infusion, he was enrolled on to our compassionate clinical protocol using haplo-TanCAR-T 19/22 cells. His father underwent apheresis, and the peripheral blood mononuclear cells (PBMCs) were used to prepare the TanCAR-T 19/22 cells. He received cytoreduction chemotherapy with vindesine $4 \mathrm{mg}$ and five daily doses of methylprednisolone $80 \mathrm{mg}$ and three daily doses of hydroxyurea $3 \mathrm{~g}$ followed by a lymphodelpeting chemotherapy with idarubicin at a total dose of $30 \mathrm{mg}$ and cyclophosphamide at a total dose of $3 \mathrm{~g}$. Planned bone marrow aspiration after the abovementioned chemotherapy and prior to haplo-TanCAR-T 19/22 cell infusion was not performed due to poor compliance of the patient. Two days later, he was treated with haploTanCAR-T 19/22 cells at a total dose of $4.72 \times 10^{6}$ TanCAR-T 19/22 cells per kilogram $\left(3.05 \times 10^{7} \mathrm{~T}\right.$ cells per kilogram, $15 \%$ transfection efficiency) administered via fractioned dosing (D0, 30\%; D1, 70\%) for safety consideration (Figs. 1 and 2).

\section{Generation of haplo-TanCAR-T 19/22 cells}

The materials and methods used in TanCAR-T 19/22 production have been described previously [14-17], with the exception of the construct of the CAR and the source of PBMCs used for manufacturing the TanCAR-T 


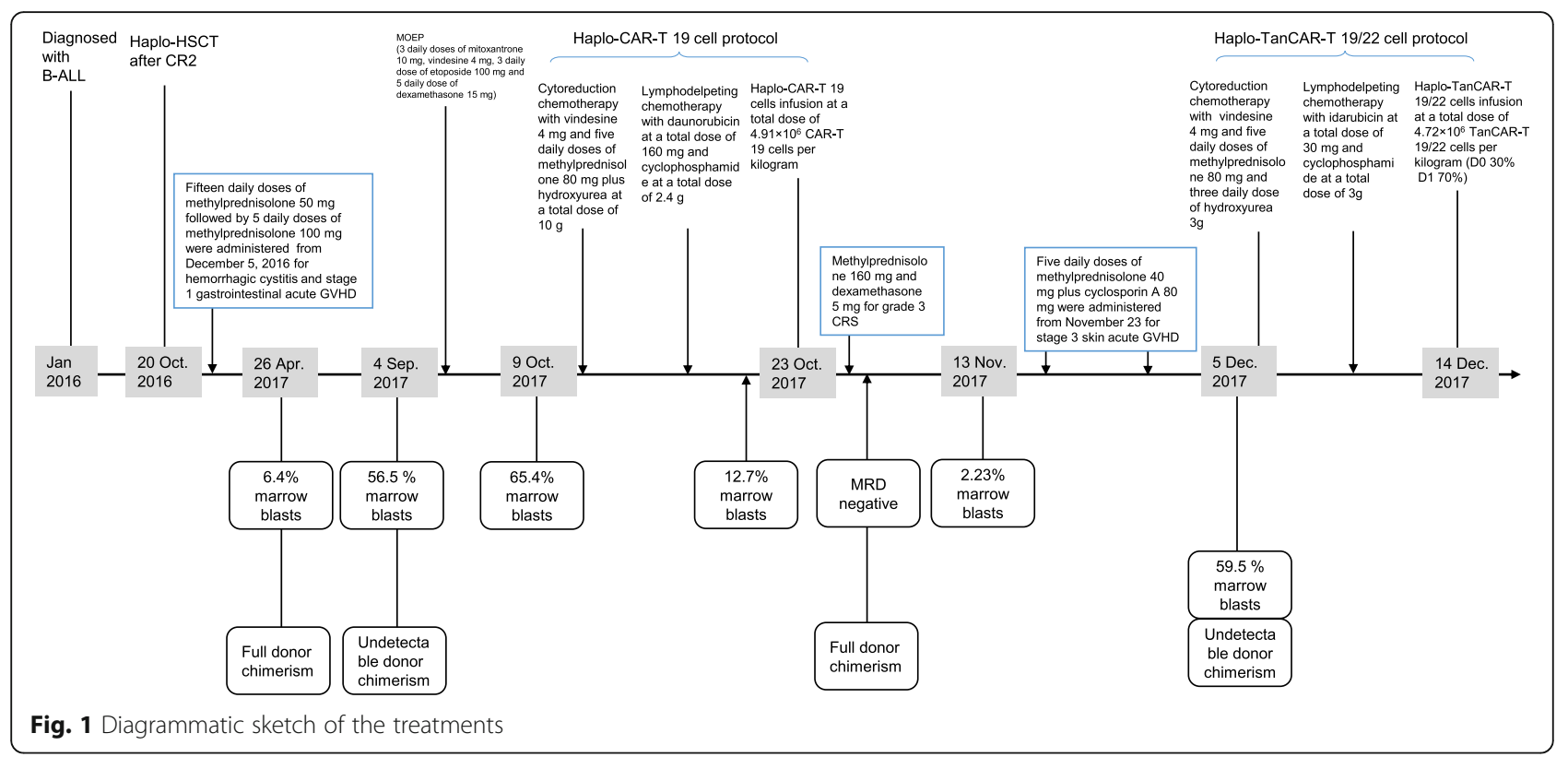

19/22 cells. TanCAR-19/22 was a tandem CAR molecule, consisting of an anti-CD22 scFv derived from mouse m971 $\mathrm{mAb}[18]$ and anti-CD19 scFv derived from the mouse FMC63 mAb [19], joined in tandem, human CD8 $\alpha$ hinge and transmembrane domain, and human CD137 and CD3 $\zeta$ signaling domains. A schematic of the TanCAR-19/22 is shown in Fig. 2a. PBMCs used for manufacturing the TanCAR-T 19/22 cells were collected by leukapheresis rather than fresh peripheral blood (PB).

\section{Detection of haplo-TanCAR-T 19/22 cells}

Flow cytometry was used for the determination of the TanCAR-19/22 transfection efficiency and quantification of the haplo-TanCAR-T 19/22 cells in clinical specimens using a Biotin-SP-AffiniPure Goat Anti-Mouse IgG, F $(\mathrm{ab})_{2}$ Fragment Specific (Jackson ImmunoResearch, USA) and PE Streptavidin antibody (BD Biosciences, USA). Haplo-TanCAR-T 19/22 cells in clinical specimens also were measured by qPCR as described [8].

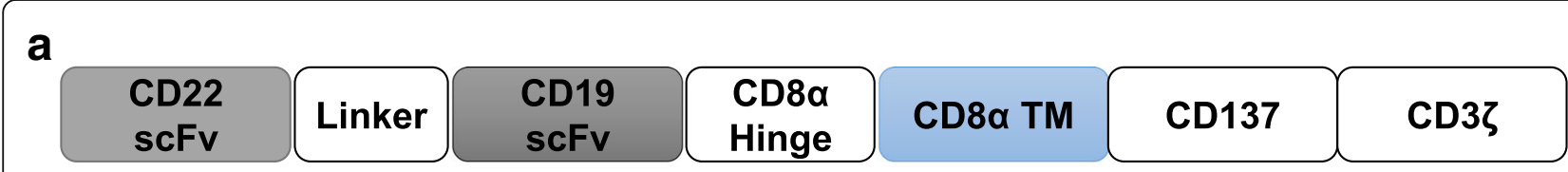

b
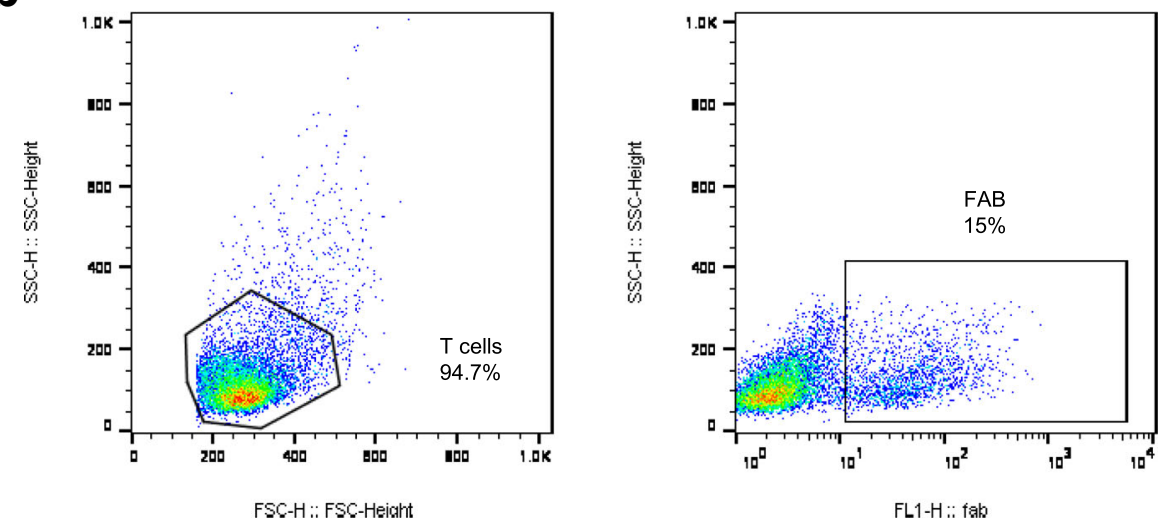

Fig. 2 Expression of the TanCAR-19/22. a Schematic of the TanCAR-19/22. b TanCAR-19/22 gene expression by FACS. As described in the "Detection of haplo-TanCAR-T 19/22 cells" section, Biotin-SP-AffiniPure Goat Anti-Mouse lgG, F (ab') 2 Fragment Specific and PE Streptavidin antibody were used 


\section{Assessment of chimerism status}

The extent of donor engraftment in clinical specimens was assessed by using short tandem repeat amplification and fluorescence labeling multiplex PCR combined with capillary electrophoresis as described [20].

\section{Cytokine measurements}

Serum interleukin (IL)-2, IL-6, IL-8, and IL-10 and tumor necrosis factor- $\alpha$ levels were batch analyzed as described [14].
Haplo-TanCAR-T 19/22 cells induced durable MRDnegative remission with full donor chimerism BM before haplo-TanCAR-T 19/22 cell protocol showed predominant blast cells with an absence of normal BM precursors. BM flow cytometry at day 14 after haploTanCAR-T 19/22 cell infusion indicated that there were $0.73 \%$ residual marrow blasts. Of note, those residual leukemic blasts exhibited the expression pattern CD34+ $\mathrm{CD} 10+\mathrm{CD} 22+\mathrm{CD} 38+\mathrm{CD} 33+\mathrm{CD} 19-\mathrm{CD} 20-$, which were undetected by flow cytometry by day 28 in the

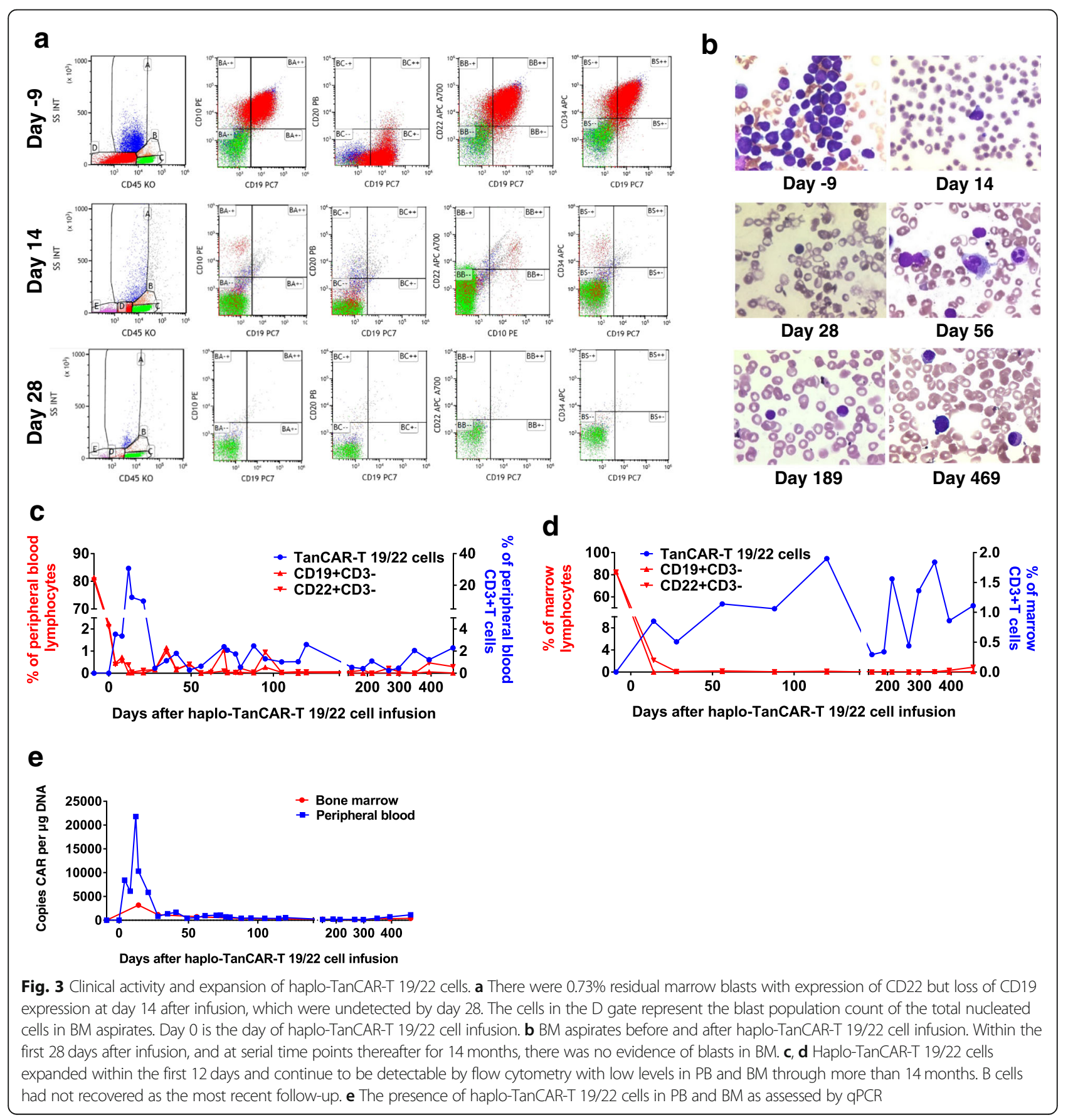


absence of further therapy (Fig. 3a). Given the incomplete recovery of platelet and absolute neutrophil count by day 28 , this patient achieved a MRD-CRi by day 28 after infusion. There was no evidence of blasts in BM either by BM smear or by flow cytometry at serial time points thereafter for 14 months (Fig. 3b and Additional file 1: Figure S1). $\mathrm{BM}$ had reconstitution of normal hematopoiesis by day 56 with the exception of platelet count that still unrecovered at a level of $36 \times 10^{9} / \mathrm{L}$ as the time of this report. Full donor chimerism was established at day 14 post infusion and remained stable thereafter.

\section{Haplo-TanCAR-T 19/22 cells exhibited substantial expansion and durable persistence in vivo}

After infusion, haplo-TanCAR-T 19/22 cells expanded and peaked at a level of $30.7 \%$ of circulating $\mathrm{T}$ cells at day 12 followed by a contraction phase with a low level of $0.45 \%$ of circulating $\mathrm{T}$ cells by day 28 . This was coincident with the elimination of circulating $\mathrm{B}$ cells that were almost undetected at day 28 by flow cytometry. Haplo-TanCAR-T 19/22 cells were still measurable with a low level of $2.29 \%$ of circulating $\mathrm{T}$ cells and the circulating $B$ cells still had not recovered as the time of this report (Fig. 3c and Additional file 1: Figure S2). HaploTanCAR-T 19/22 cells were also present by flow cytometry at all the response evaluation time points in $\mathrm{BM}$ obtained at response evaluation, and chronic B cell aplasia was documented (Fig. 3d and Additional file 1: Figure S2). An overall concordance between the expansion and persistence of haplo-TanCAR-T 19/22 cells in PB measured by flow cytometry and qPCR was observed. As the time of this report, TanCAR-19/22 DNA remained detectable on qPCR with 1134 and 396 copies per microgram DNA in $\mathrm{PB}$ and $\mathrm{BM}$, respectively (Fig. 3e).

\section{Toxicity following haplo-TanCAR-T 19/22 cell infusion CRS}

After haplo-TanCAR-T 19/22 cell infusion, he experienced grade 3 CRS graded according to the UPenn grading scale $[21,22]$. Fever of up to $38.8^{\circ} \mathrm{C}$ occurred within $24 \mathrm{~h}$ after haplo-TanCAR-T 19/22 cell infusion, lasting 11 days and becoming afebrile at day 12 after treated with a lower dose of tocilizumab at $160 \mathrm{mg}(1.6 \mathrm{mg} / \mathrm{kg})$ and etanercept $50 \mathrm{mg}$ at day 8 (Fig. 4a). Multiple serum cytokines had markedly increased 7 days post infusion and almost returned to baseline values by day 41 (Fig. 4b, c), where interleukin (IL)-6 levels peaked at $3377 \mathrm{pg} / \mathrm{mL}$ (88-fold over baseline) at day 11. Aspartate aminotransferase and lactate dehydrogenase significantly elevated 8

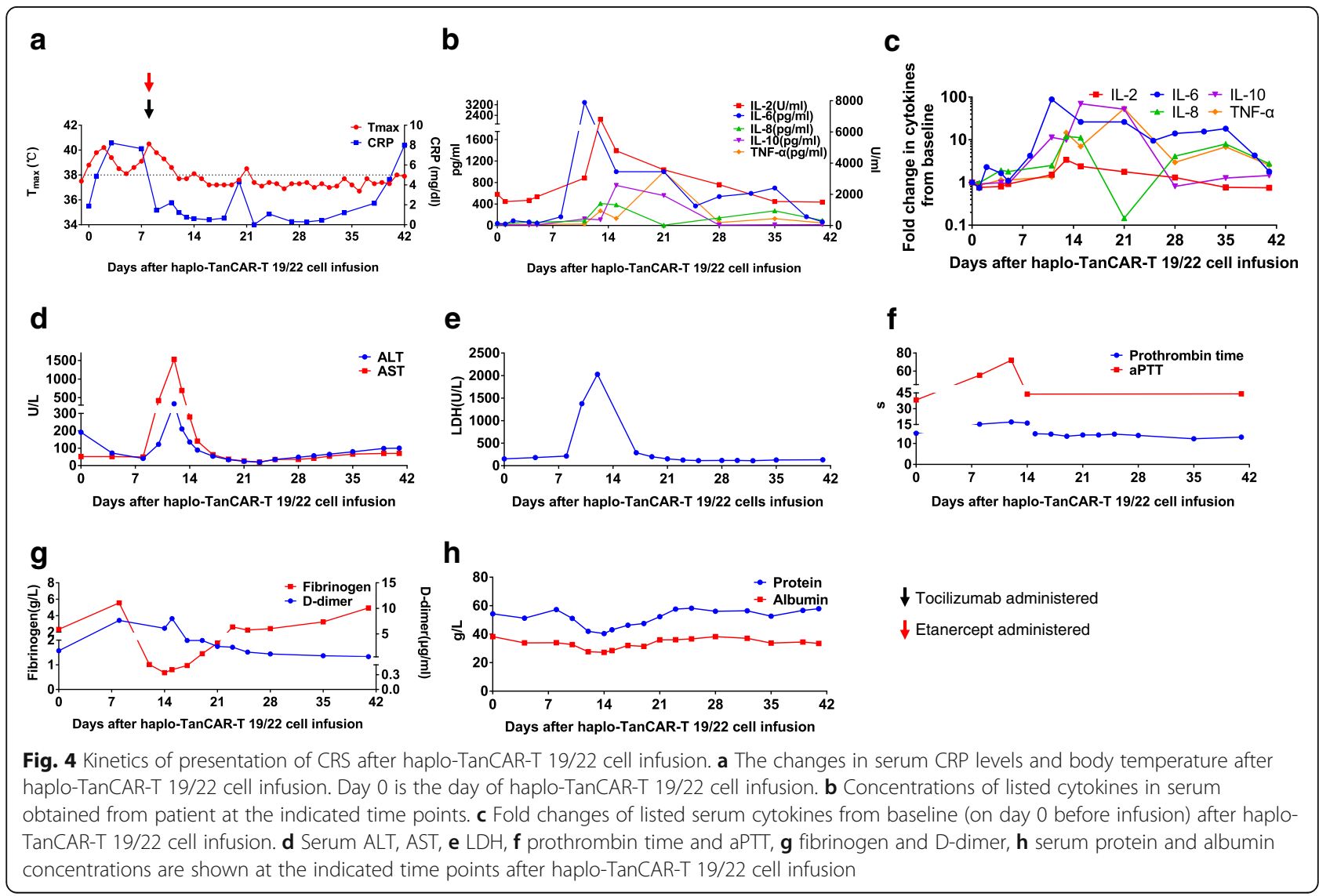


to 10 days after infusion, peaked at $1529.1 \mathrm{U} / \mathrm{L}$ (38-fold over upper limit of normal) and 2027.8 U/L (13-fold over baseline) at day 12, respectively, and returned to baseline values by day 21 with best support care (Fig. $4 \mathrm{~d}$, e). He also exhibited coagulation dysfunction with prolonged activated partial thromboplastin time, elevated D-dimer, and fallen fibrinogen concentrations, as well as capillary leak with grade 2 hypoalbuminemia in spite of intensive supplementation of protein during the CRS, which resolved by day 23 (Fig. 4f-h).

\section{GVHD}

The prior stage 3 skin acute GVHD that was under control was reactivated and rapidly progressed to stage 4 skin GVHD with new-onset local skin ulcerations particularly in the scrotal skin and mouth mucosa 11 days after haplo-TanCAR-T 19/22 cell infusion (Fig. 5a). The concentration of serum total bilirubin continually elevated from day 12 and increased to $134 \mu \mathrm{mol} / \mathrm{L}$ at day 21 (Fig. 5b). Given the rapidly progressive skin GVHD manifestations and liver involvement, lower-dose methylprednisolone at $20 \mathrm{mg}$ daily as the initial dose with subsequent tapering in an effort to balance the benefits and risks of systemic immunosuppression was implemented from day 21 and discontinued by day 39. Skin rash and serum total bilirubin improved significantly after those treatments. However, stage 3 gut GVHD manifestations mainly including diarrhea occurred from day 50 , and the serum total bilirubin elevated again, suggesting a grade 3 acute GVHD. Sixteen doses of methylprednisolone $20 \mathrm{mg}$ per day were administered again from day 78, significantly controlling diarrhea and serum total bilirubin. This patient subsequently exhibited moderate chronic GVHD mainly manifested as scleroderma, diarrhea, and weight loss. Persistent thrombocytopenia with platelet count ranging from $15 \times 10^{9}$ to $43 \times 10^{9} / \mathrm{L}$ without platelet transfusion could be acknowledged as a manifestation of chronic GVHD in the setting of reconstitution of normal hematopoiesis. The systemic immunosuppressive treatment was tapered within 2 months with methylprednisolone $4 \mathrm{mg}$ every other day and methotrexate $5 \mathrm{mg}$ once a week and sirolimus $1 \mathrm{mg}$ daily as a minimum maintenance dose from day 154 to the time of this report (Fig. 5b), keeping the chronic GVHD under good control.

\section{Discussion and conclusions}

We report an adult patient who had rapidly progressive leukemia after haplo-HSCT with overwhelming disease burden at baseline obtained MRD-CR continuing for more than 14 months with manageable GVHD by taper

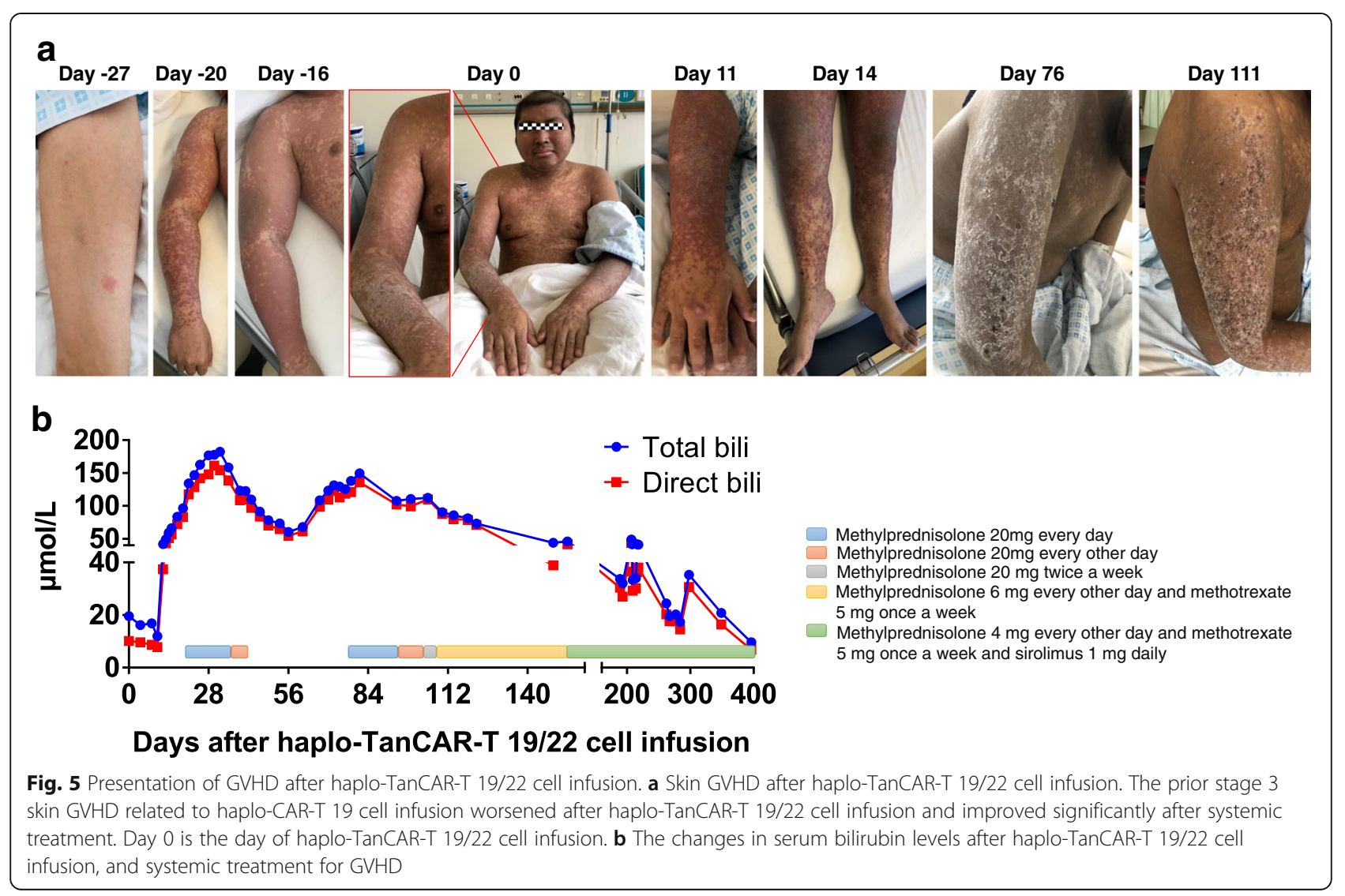


schedule after treated on haplo-TanCAR-T 19/22 cell protocol. This sustained remission duration could be comparable to that of Novartis's CTL019 in pivotal ELIANA phase II trial, where the rate of relapse-free survival among 61 patients with a response to treatment was $80 \%$ at 6 months and $59 \%$ at 12 months, and most of the relapsed patients had CD19-negative disease [23].

Three mechanisms, direct antimalignancy activity of cytoreduction chemotherapy and lymphodepleting chemotherapy, graft-versus-leukemia (GVL) effect mainly mediated by the donor $\mathrm{T}$ cells contained in the graft, and targeted immune rejection of leukemia cells with expression of CD19 and/or CD22 by TanCAR-T 19/22 cells, could contribute to the eradication and sustained control of leukemia cells in this case. Among those anti-tumor factors, the key driving force involved in the induction of sustained remission should most possibly be attributed to the TanCAR-T 19/22 cell-mediated specific killing rather than the nonspecific anti-tumor activities raised from GVL effect and/or chemotherapy according to the following characteristics of clinical course of this patient: (1) HaploCAR-T 19 cell protocol and haplo-TanCAR-T 19/22 cell protocol were highly similar (as shown in Fig. 1), with the notable exception of in vivo persistence of infused CAR-T cells. Sustained remission was only achieved after infusion of haplo-TanCAR-T 19/22 cells with prolonged persistence rather than haplo-CAR-T 19 cells with transient persistence probably due to unknown abnormal early expansion and steroid use for treatment of sCRS following haploCAR-T 19 cell infusion. (2) Prolonged B cell aplasia was observed in this patient. Although the cytoreduction chemotherapy and lymphodepleting chemotherapy also were expected to induce B cell aplasia, but sustained B cell aplasia for more than 14 months with recovery of other blood cell counts seen in this patient only was related to the continued specific immunosurveillance provided by the low level of persisting haplo-TanCAR-T 19/22 cells as shown in Additional file 1: Figure S3. (3) The patient achieved CR by day 14 and MRD-CR by day 28 after haplo-TanCAR-T 19/22 cell infusion, which had been reported in clinical trials of CD19-targeted CAR-T cell for R/R B-ALL [24-26], while the remissions to standard donor lymphocyte infusion, a successful embodiment of GVL effect, were rare with reported CR rates of 0 to $25 \%$ [27], and the onset of remission typically occurred over several weeks. Furthermore, the dose of haploidentical $\mathrm{CD} 3+$ cells this patient received was one magnitude lower than that of the standard donor lymphocyte infusion dose. Therefore, the initial remission due to the GVL effect for this patient who relapsed after haplo-HSCT was less likely. Collectively, those observations highlighted that TanCART 19/22 cell-mediated specific killing was primarily responsible for the continued remission of this patient. But it must be emphasized that the persistent allogeneic $\mathrm{T}$ cell responses suggested by the sustained chronic GVHD could not be ruled out for contributing to the long-term disease control albeit his disease had relapsed after haploHSCT in the case of full donor chimerism. Moreover, haplo $T$ cells rather than haplo-TanCAR-T 19/22 cells had advantages in controlling the evolution of CD19- and CD22-double escape variants or clonally related malignancies in other lineages. It will be clearer how TanCAR-T $19 / 22$ cells contribute to the long-term disease control in our well-designed clinical trial of autologous TanCAR-T 19/22 cells in R/R B-ALL.

The main safety concern for this patient after haploTanCAR-T 19/22 cell infusion could be the increased risk of the recrudescence of the prior haplo-CAR-T 19 cell infusion-related GVHD that was under control prior to haplo-TanCAR-T 19/22 cell infusion. Not surprisingly, the patient developed grade 3 acute GVHD within 2 months after haplo-TanCAR-T 19/22 cell infusion. How to balance the benefits and risks of systemic immunosuppression was a unique challenge for physician to management of the GVHD this patient experienced. Methylprednisolone at $2 \mathrm{mg} / \mathrm{kg} /$ day as the initial dose followed by tapering dose after initial response had been accepted as a standard firstline systemic therapy for acute GVHD [28]. Apparently, this initial dose of methylprednisolone would greatly increase the risk of mediating a greater adverse effect on the antitumor activity of haplo-TanCAR-T 19/22 cells; thus, we used a lower-dose methylprednisolone at $20 \mathrm{mg}$ daily as the initial dose followed by elegant titratable dosing in an effort to partially treat GVHD or slow down the GVHD exacerbation but without impair the anti-tumor activity of haploTanCAR-T 19/22 cells. The fact indicated that this strategy worked well and reached the effect to be hoped. As observed in this case, steroid exposure would become more frequent and even inevitable in the case of the onset of GVHD; in addition, CRS was always accompanied by acute GVHD in the case of donor-derived CAR-T cells [9], and conditions were difficult to distinguish, making the steroid use more challenging. Here, we established a practical way to titrate GVHD and anti-tumor activity of CAR-T cells, whereby the short-term and long-term clinical response was not affected. It could make sense not only for the management of the GVHD and/or sCRS related to donor-derived CAR-T cells, but also for early immunomodulation for the prevention of severe neurotoxicity.

This application of haplo-TanCAR-T 19/22 cells has provided a demonstration of the potential of inducing durable remission of R/R B-ALL by CAR simultaneously targeting CD19 and CD22, albeit with a clinical experience limited to one case. Moreover, allogeneic CAR therapy in the posttransplant setting potentially confounds the role of TanCAR-T 19/22 cells for this continued remission. Anyway, this finding should encourage 
continued study of this product, and actually, the welldesigned clinical trial of autologous TanCAR-T 19/22 cells in adult patients with R/R B-ALL is now under way. In addition, although second infusion of haploCAR-T cells has succeeded in this case, it should be cautioned in other patients particularly in those patients with prior GVHD and must be evaluated case by case.

\section{Additional file}

Additional file 1: Figure S1. Bone marrow immunophenotyping at serial time points after haplo-TanCAR-T 19/22 cell infusion. There was no evidence of blasts in BM at day 56 and serial time points thereafter for 14 months.

Figure S2. Prolonged B cell aplasia after haplo-TanCAR-T 19/22 cell infusion. $B$ cells were eliminated from $P B$ and $B M$ and had not recovered more than 1 year after haplo-TanCAR-T 19/22 cell infusion. Day 0 is the day of haploTanCAR-T 19/22 cell infusion. B cells were measured by flow cytometry for CD19 and CD22. Figure S3. CD22-specific immunosurveillance mediated by haplo-TanCAR-T 19/22 cells. The circulating CD22+CD19- B cell subclones accounting for $0.56 \%$ of circulating lymphocytes at day 95 were undetected by day 105. Day 0 is the day of haplo-TanCAR-T 19/22 cell infusion. B cells were measured by flow cytometry for CD19 and CD22. Figure S4. CD19 and CD22 marker expression in BM before haplo-CAR-T 19 cell infusion and haplo-TanCAR-T 19/22 cell infusion. The cells in the D gate represent the blast population count of the total nucleated cells in BM aspirates. (PPTX $3084 \mathrm{~kb})$

\section{Abbreviations}

B-ALL: B cell acute lymphoblastic leukemia; BM: Bone marrow; CAR$T$ : Chimeric antigen receptor T; CR: Complete remission; CRi: CR with incomplete count recovery; GVHD: Graft versus host disease; GVL: Graftversus-leukemia; haplo: Haploidentical; HSCT: Hematopoietic stem cell transplantation; IL: Interleukin; MRD: Minimal residual disease; MRD-CR: MRDnegative CR; PB: Peripheral blood; PBMCs: Peripheral blood mononuclear cells; SCRS: Severe CRS

\section{Acknowledgements}

Not applicable.

\section{Authors' contributions}

HJJ conducted the study, provided patient care, analyzed the data, and wrote the paper. ZGW analyzed the data and was a major contributor in writing the manuscript. YW produced clinical cell products and analyzed the data. YL participated in the clinical care, supervised the research, and analyzed the data. HRD, $C T$, and YLG participated in the generation of clinical cell products and performed flow cytometry. BG participated in the clinical care and analyzed the data. DDT and XH participated in the generation of clinical cell products and performed QPCR. QMY participated in the clinical care, supervised the research, and analyzed the data. ZQW produced the clinical gene therapy vector and analyzed the data. WDH conceived of the study, participated in the clinical care, supervised the research, and reviewed the manuscript. All authors read and approved the final manuscript.

\section{Funding}

This research was supported by the grants from the National Natural Science Foundation of China (No. 81830002 and 31870873 to WDH) and the National Key Research and Development Program of China (No. 2016YFC1303501 and 2016YFC1303504 to WDH and No. 2017YFC0909803 to YW) and Beijing Nova Program (Z161100004916128 to YJZ).

\section{Availability of data and materials}

The datasets supporting the conclusions of this article are included in this published article and its supplementary information files.

\section{Ethics approval and consent to participate}

Ahead of planned phase I clinical trials, TanCAR-T 19/22 cells were under compassionate use for this patient. The treatment for this patient was provided under the Declaration of Helsinki and approval of the Institutional Review Board at the Chinese PLA General Hospital and with informed consent.

\section{Consent for publication}

Written informed consent was obtained from the patient.

\section{Competing interests}

The authors declare that they have no competing interests.

\section{Author details}

${ }^{1}$ Molecular \& Immunological Department, Bio-therapeutic Department, Chinese PLA General Hospital, No. 28 Fuxing Road, Beijing 100853, China. ${ }^{2}$ Department of Geriatric Hematology, Chinese PLA General Hospital, No. 28 Fuxing Road, Beijing 100853, China.

Received: 27 February 2019 Accepted: 10 May 2019

Published online: 10 June 2019

\section{References}

1. Wang Z, Wu Z, Liu Y, Han W. New development in CAR-T cell therapy. J Hematol Oncol. 2017;10:53.

2. Majzner RG, Mackall CL. Tumor antigen escape from CAR T-cell therapy. Cancer Discov. 2018;8:1219-26.

3. Fry TJ, Shah NN, Orentas RJ, Stetler-Stevenson M, Yuan CM, Ramakrishna S, et al. CD22-targeted CAR T cells induce remission in B-ALL that is naive or resistant to CD19-targeted CAR immunotherapy. Nat Med. 2018;24:20-8.

4. Schultz LM, Davis KL, Baggott C, Chaudry C, Marcy AC, Mavroukakis S, et al. Phase 1 study of CD19/CD22 bispecific chimeric antigen receptor (CAR) therapy in children and young adults with B cell acute lymphoblastic leukemia (ALL). Blood. 2018;132:898.

5. Hossain N, Sahaf B, Abramian M, Spiegel JY, Kong K, Kim S, et al. Phase I experience with a bi-specific CAR targeting CD19 and CD22 in adults with B-cell malignancies. Blood. 2018;132:490.

6. Gardner R, Annesley C, Finney O, Summers C, Lamble AJ, Rivers J, et al. Early clinical experience of CD19 × CD22 dual specific CAR T cells for enhanced anti-leukemic targeting of acute lymphoblastic leukemia. Blood. 2018;132:278.

7. Amrolia PJ, Wynn R, Hough R, Vora A, Bonney D, Veys P, et al. Simultaneous targeting of CD19 and CD22: phase I study of AUTO3, a bicistronic chimeric antigen receptor (CAR) T-cell therapy, in pediatric patients with relapsed/refractory B-cell acute lymphoblastic leukemia ( $r / r$ B-ALL): Amelia Study. Blood. 2018;132:279.

8. Dai H, Zhang W, Li X, Han Q, Guo Y, Zhang Y, et al. Tolerance and efficacy of autologous or donor-derived T cells expressing CD19 chimeric antigen receptors in adult B-ALL with extramedullary leukemia. Oncoimmunology. 2015:4:e1027469.

9. Chen Y, Cheng Y, Suo P, Yan C, Wang Y, Chen Y, et al. Donor-derived CD19targeted $\mathrm{T}$ cell infusion induces minimal residual disease-negative remission in relapsed B-cell acute lymphoblastic leukaemia with no response to donor lymphocyte infusions after haploidentical haematopoietic stem cell transplantation. Br J Haematol. 2017;179:598-605.

10. Kochenderfer JN, Dudley ME, Carpenter RO, Kassim SH, Rose JJ, Telford WG, et al. Donor-derived CD19-targeted T cells cause regression of malignancy persisting after allogeneic hematopoietic stem cell transplantation. Blood. 2013;122:4129-39.

11. Brudno JN, Somerville RP, Shi V, Rose JJ, Halverson DC, Fowler DH, et al. Allogeneic $T$ cells that express an anti-CD19 chimeric antigen receptor induce remissions of B-cell malignancies that progress after allogeneic hematopoietic stem-cell transplantation without causing graft-versus-host disease. J Clin Oncol. 2016;34:1112-21.

12. Liu J, Zhong JF, Zhang X, Zhang C. Allogeneic CD19-CAR-T cell infusion after allogeneic hematopoietic stem cell transplantation in B cell malignancies. J Hematol Oncol. 2017;10:35.

13. Anwer F, Shaukat AA, Zahid U, Husnain M, McBride A, Persky D, et al. Donor origin CAR T cells: graft versus malignancy effect without GVHD, a systematic review. Immunotherapy. 2017;9:123-30.

14. Wang Y, Chen M, Wu Z, Tong C, Dai H, Guo Y, et al. CD133-directed CAR T cells for advanced metastasis malignancies: a phase I trial. Oncoimmunology. 2018;7:e1440169. 
15. Guo Y, Feng K, Liu Y, Wu Z, Dai H, Yang Q, et al. Phase I study of chimeric antigen receptor-modified T cells in patients with EGFR-positive advanced biliary tract cancers. Clin Cancer Res. 2018;24:1277-86.

16. Wang $\mathrm{CM}, \mathrm{Wu} Z \mathrm{ZQ}$, Wang $\mathrm{Y}$, Guo $\mathrm{YL}$, Dai HR, Wang $\mathrm{XH}$, et al. Autologous T cells expressing CD30 chimeric antigen receptors for relapsed or refractory Hodgkin lymphoma: an open-label phase I trial. Clin Cancer Res. 2017;23:1156-66.

17. Zhang WY, Wang Y, Guo YL, Dai HR, Yang QM, Zhang YJ, et al. Treatment of CD20-directed chimeric antigen receptor-modified $T$ cells in patients with relapsed or refractory B-cell non-Hodgkin lymphoma: an early phase lla trial report. Signal Transduct Target Ther. 2016;1:16002

18. Xiao X, Ho M, Zhu Z, Pastan I, Dimitrov DS. Identification and characterization of fully human anti-CD22 monoclonal antibodies. mAbs. 2009;1:297-303.

19. Kochenderfer JN, Feldman SA, Zhao Y, Xu H, Black MA, Morgan RA, et al: Construction and preclinical evaluation of an anti-CD19 chimeric antigen receptor. J Immunother. 2009;32:689-702.

20. Li SX, Zhu HL, Guo B, Da WM. Application of short-tandem-repeat amplification and fluorescent-multiplex PCR for chimerism analysis. Zhongguo Shi Yan Xue Ye Xue Za Zhi. 2011;19:749-53.

21. Porter D, Frey N, Wood PA, Weng Y, Grupp SA. Grading of cytokine release syndrome associated with the CAR T cell therapy tisagenlecleucel. J Hematol Oncol. 2018;11:35

22. Liu D, Zhao J. Cytokine release syndrome: grading, modeling, and new therapy. J Hematol Oncol. 2018;11:121.

23. Maude SL, Laetsch TW, Buechner J, Rives S, Boyer M, Bittencourt H, et al. Tisagenlecleucel in children and young adults with B-cell lymphoblastic leukemia. N Engl J Med. 2018;378:439-48.

24. Turtle CJ, Hanafi LA, Berger C, Gooley TA, Cherian S, Hudecek M, et al. CD19 CAR-T cells of defined CD4+:CD8+ composition in adult B cell ALL patients. J Clin Invest. 2016;126:2123-38.

25. Gardner RA, Finney O, Annesley C, Brakke H, Summers C, Leger K, et al. Intentto-treat leukemia remission by CD19 CAR T cells of defined formulation and dose in children and young adults. Blood. 2017;129:3322-31.

26. Brentjens RJ, Davila ML, Riviere I, Park J, Wang X, Cowell LG, et al. CD19-targeted $T$ cells rapidly induce molecular remissions in adults with chemotherapy-refractory acute lymphoblastic leukemia. Sci Transl Med. 2013;5:177ra138

27. Shiobara S, Nakao S, Ueda M, Yamazaki H, Takahashi S, Asano S, et al. Donor leukocyte infusion for Japanese patients with relapsed leukemia after allogeneic bone marrow transplantation: lower incidence of acute graft-versus-host disease and improved outcome. Bone Marrow Transplant. 2000;26:769-74.

28. Martin PJ, Rizzo JD, Wingard JR, Ballen $\mathrm{K}$, Curtin PT, Cutler $\mathrm{C}$, et al. First- and second-line systemic treatment of acute graft-versus-host disease: recommendations of the American Society of Blood and Marrow Transplantation. Biol Blood Marrow Transplant. 2012;18:1150-63.

Ready to submit your research? Choose BMC and benefit from:

- fast, convenient online submission

- thorough peer review by experienced researchers in your field

- rapid publication on acceptance

- support for research data, including large and complex data types

- gold Open Access which fosters wider collaboration and increased citations

- maximum visibility for your research: over $100 \mathrm{M}$ website views per year

At BMC, research is always in progress.

Learn more biomedcentral.com/submissions 